\title{
A Group Decision Approach for Supplier Selection Problem Based on a Multi-criteria Model
}

\author{
Constanţa Zoie RĂDULESCU ${ }^{1 *}$, Marius RĂDULESCU ${ }^{2}$ \\ ${ }^{1}$ National Institute for Research and Development in Informatics, 8-10, Maresal Averescu Avenue, \\ Bucharest, 01145, Romania \\ zoie.radulescu@ici.ro (*Correspondingauthor) \\ 2 "Gheorghe Mihoc-Caius Iacob" Institute of Mathematical Statistics and Applied Mathematics, \\ Calea 13 Septembrie No.13, Bucharest, 050711, Romania \\ mradulescu.csmro@yahoo.com
}

\begin{abstract}
The aim of this paper is to develop a group decision approach for the supplier selection problem. Suppose that a manager wants to purchase a set of devices based on the offer of a few suppliers such that several constraints are met. The proposed decision support approach combines a group decision method that calculates the performance devices and the selection risk associated with it, as well as a Multi-Objective model for the supplier selection. Every single device is evaluated by a group of evaluators across a certain set of criteria. The divergent opinions of the evaluators generate the selection risk related to the respective portfolio of devices. A new Multi-Objective model for the supplier selection problem is formulated. It has two objective functions: risk minimization and performance maximization. Starting from the aforementioned model a minimum risk model with budget and performance constraints has been formulated. A case study connected with the purchase of certain medical devices has been conducted and the related numerical results have been analysed. Our approach can help managers in the purchasing management. Based on it a software module can be realized and embedded in a decision support system.
\end{abstract}

Keywords: Supplier selection problem, Group decision approach, Multi-objective, Risk minimization model, Weights.

\section{Introduction}

Supplier selection is a complex process which requires:

- evaluation of several suppliers across several criteria;

- selection of suppliers that are able to provide the buyer with products and/or services that meet his requirements. The requirements include quality standards of the buyer, the budget constraints, the quantities that describe the demand constraints and the delivery constraints.

Decision techniques are an important part of the supplier selection problem and contribute to the development of decision support applications. Decision-making is a specific form of information processing that aims at setting-up an action plan under specific circumstances (Filip et al., 2017).

In supply chain literature, supplier evaluation and selection problem are one of the most systematically analyzed subjects as this issue is important for maintaining the sustainability and profitability of the supply chain. This paper sets out to solve the supplier selection problem by means of a group decision approach.

This paper is organized as follows. Section 2 includes a literature review on the methods applied for solving supplier selection problem. Section 3 is devoted to the formulation of a group decision approach with two stages. In the first stage the suppliers are evaluated, the performance of the devices and the associated selection risk are calculated. The idea of evaluating the devices out of suppliers' offer is inspired from financial portfolio theory. The divergent opinions of evaluators generate the selection risk related to the aforementioned portfolio of devices. The higher the divergences among the evaluators`opinions, the higher the selection risk becomes.

In the second stage the supplier selection problem is solved based on a new Multi-Objective optimization model.

In the fourth section a case study for a healthcare centre is analyzed. The final section is dedicated to general conclusions.

\section{Literature Review}

Dickson in (Dickson, 1966) and Weber in (Weber et al., 1991) made researches in the criteria for supplier selection trying to rank the importance for each criterion. In the above-mentioned research works, quality, delivery performance and purchasing cost are regarded as the main criteria for supplier selection. In real-life situations there are more than 
three criteria for supplier selection. The ranking of those criteria depends on buyers' preferences.

The papers (de Boer et al., 2001), (Chai et al., 2013), (Degraeve et al., 2000), (Ho et al., 2010), (Weber et al., 1991) provide comprehensive literature reviews on supplier evaluation and selection problem. In the last years several reviews have been carried out for the specialized literature on the problem of supplier selection.

(Zimmer et al., 2016), (Fahimnia et al., 2015), (Wetzstein et al., 2016). A recent review of the most relevant papers on the aforementioned topic published between 2013 and 2018 was made by (Chai \& Ngai, 2020).

Various decision-making approaches have been proposed to solve the supplier selection problem.

Operations research provides a wide range of methods and techniques that may support the buyer in the purchasing decision. More precisely, it helps the buyer deal with increased complexity of the procurement process.

Examples of such methods are the multi-criteria decision-making techniques, mathematical programming, artificial intelligence and data mining techniques.

Mathematical programming methods have been widely used for solving the supplier selection problem. Usually mathematical programming models have several objectives such as cost, performance and risk. In case that a single criterion is taken into account most of the models consider the cost criterion.

In case that several objectives are taken into account one can transform the initial problem.

Into a single-objective problem whose aim would be a linear combination of the initial objectives. This can be done with the help of some weights that show the importance of each of the objectives in the selection process. The respective weights may be determined by using certain multi-criteria weighting methods. See for example (Baykasoglu et al., 2013) for Decision Making Trial and Evaluation Laboratory - DEMATEL method, (Kersuliene et al., 2010) for Stepwise Weight Assessment Ratio Analysis SWARA method, (Rădulescu \& Rădulescu, 2018) for a review for weighing multi-criteria methods, (Rezaei, 2015) for Best-worst multi-criteria decision-making method, (Saaty, 1980) for Analytic Hierarchy Process, (Zavadskas et al., 2019) and (Zolfani et al., 2018) for Extended Stepwise Weight Assessment Ratio Analysis (E-SWARA) method.

Further on, several papers that make use of mathematical programming are shortly reviewed.

In (Sodenkamp et al., 2016) a novel meta-approach for supporting collaborative Multi-Objective supplier selection and order allocation decisions was proposed. The respective approach integrates multi-criteria decision analysis and linear programming. It incorporates both heterogeneous objective data and subjective judgments of the decision makers representing various groups with different voting powers.

In (Hu et al., 2016) a mixed integer programming model is proposed to solve the supplier selection and order allocation problem for a manufacturer. In the framework of this model, quality, delivery performance, and purchasing cost are chosen as the three objectives related to the supplier selection process. Inventory level, goods low balance, service level, ability to supply, and marketing demand are considered to be constraints. In (Kellner \& Utz, 2019) a MultiObjective optimization model that is based on the financial portfolio theory is formulated. This model transfers the traditional risk-expected return tradeoff to the supplier selection and order allocation context and extends the classical portfolio model through by adding objective functions and constraints with integer variables. The objectives of the aforementioned model are: cost, sustainability and supply risk. Its constraints are: demand satisfaction, supplier capacity, minimum order quantity, budget, logistics service, dual sourcing, maximum number of suppliers, number of strategic suppliers and number of regional suppliers.

The supplier selection problem may include several uncertainties which are related with suppliers. In (Dupont et al., 2018) supplier selection is analyzed while taking into account the risk of delivery failure. Suppliers are located in different geographic areas, and supplies are subject to a positive probability of disruption. Different capacity and failure probabilities for each supplier are considered. The authors use a mixed-integer linear programming approach in order to provide decision-making support. 


\section{A Group Decision Approach for Supplier Selection}

Assume a manager wants to buy certain devices and select from the suppliers' offer a portfolio of devices that indicates his preferences. When making a choice the manager has two objectives: to maximize the performance of those devices and to minimize the inherent selection risk. This is a multi-criteria group decision problem because several evaluators are involved in the selection of devices portfolio.

The decision approach has two stages. In the first stage the supplier selection problem is defined and a method based on group decision is developed which consists in evaluating the offer of several suppliers across several criteria.

The performance of the aforementioned devices is evaluated by a group of evaluators that assign scores to each device belonging to a supplier based on a certain set of criteria. The quality of the expertise of each evaluator is considered based on the coefficient of authority. An overall performance of those devices is defined with regard to the criteria weights. In this stage one calculates the performance of the devices and the inherent selection risk. These are the inputs in the second stage of the approach.

In the second stage a new Multi-Objective optimization model for the supplier selection is formulated. This model has two objective functions: risk minimization and performance maximization. Starting from this model a minimum risk model is formulated. Several constraints such as budget constraints and performance constraints are included in the model. Two parameters: sum to invest and lower limit of the performance are also included. A very important aspect when solving the supplier selection problem is determining the related range of parameters. A sensitivity analysis is performed for the respective range of parameters. For each of those parameters a minimum and a maximum optimization model is solved.

The group decision approach for supplier selection is illustrated in Figure 1.

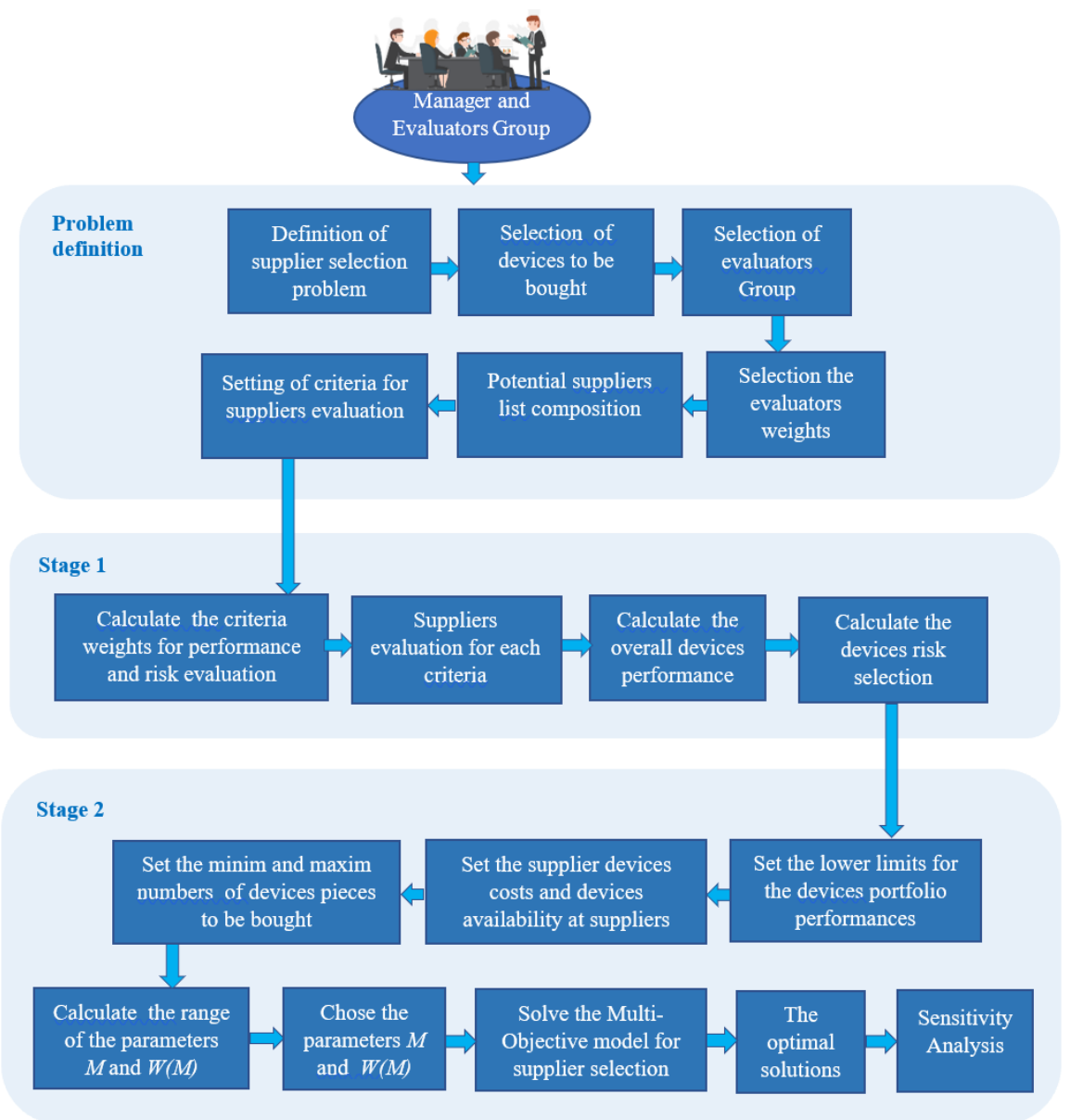

Figure 1. The group decision approach for supplier selection 


\subsection{The Devices Performance and the Selection Risk of Devices}

In stage 1 of the group decision approach the device performance and the related selection risk are calculated based on a multi-attribute group decision making method.

The input data in stage 1 is included in Table 1.

The group of evaluators selects the criteria for the evaluation of devices and the related measurement scale. Each evaluator evaluates each device with regard to each of the above-mentioned criteria and for each supplier. The related scores are $s_{i, j, \alpha, \beta}$.

The weights involved in the aforementioned model are:

(a) $w_{1, \alpha, \beta}$ - the importance of the evaluation made by evaluator $\beta$ for criterion $\alpha$ (coefficient of authority);

(b) $w_{2, \alpha}=$ the importance of criterion $\alpha$ in the performance evaluation process;

(c) $w_{3, \alpha}=$ the importance of criterion $\alpha$ in the risk evaluation process.

They can be obtained using several multicriteria methods.

Suppose that $w_{1, \alpha, \beta}$ for all $\alpha, \beta$ and

$\sum_{\boldsymbol{\beta}=1}^{\boldsymbol{q}} w_{1, \boldsymbol{\alpha}, \boldsymbol{\beta}}$ for all $\alpha$.

Suppose that $w_{2, \dot{a}} \geq 0$. for all $\alpha$ and

$\sum_{\alpha=1}^{q} w_{2, \alpha}=1$.

Suppose that $w_{3, \dot{a}} \geq 0$ for all $\alpha$ and

$\sum_{\alpha=1}^{q} w_{3, \alpha}=1$
Calculate the performance according criterion $\alpha$ of devices of type $i$ sold by supplier $j$ :

$\mu_{i, j, \alpha}=\sum_{\beta=1}^{q} w_{1, \alpha, \beta} S_{i, j, \alpha, \beta}$

Calculate the performance of devices of type $i$ sold by supplier $j$.

$p_{i, j}=\sum_{\alpha=1}^{r} w_{2, \alpha} \sum_{\beta=1}^{q} w_{1, \alpha, \beta} S_{i, j, \alpha, \beta}$

$i=1,2, \ldots, n ; j=1,2, \ldots, m$

Calculate the selection risk for the devices involved according to criterion $\alpha$ related to the devices of type $i$ sold by supplier $j$.

$$
\begin{gathered}
v_{i, j, \alpha}=\sum_{\beta=1}^{q} w_{1, \alpha, \beta}\left(s_{i, j, \alpha, \beta}-\mu_{i, j, \alpha}\right)^{2} \\
i=1,2, \ldots, n ; j=1,2, \ldots, m, \alpha=1,2, \ldots, r
\end{gathered}
$$

Calculate the risk of selection devices of type $i$ sold by supplier $j$.

$\rho_{i, j}=\sum_{\alpha=1}^{r} w_{3, \alpha} v_{i, j, \alpha}$

The performance of devices $p_{i, j}$ and the selection risk for devices $\rho_{i, j}$ are inputs in stage 2 of the group decision approach.

\subsection{The Multi-Objective Optimization Model for Supplier Selection}

In stage 2 of the decision approach an original Multi-Objective optimization model for supplier selection is formulated.

The input data in stage 2 are included in Table 2.

The decision variable of the Multi-Objective model for supplier selection is $\mathbf{x}=\left(x_{i j}\right), i=1,2, \ldots, n$;

Table 1. Input data in stage 1 the decision approach

\begin{tabular}{|l|l|}
\hline Symbol & Description \\
\hline$n$ & Number of devices to be purchased \\
\hline$m$ & Number of suppliers \\
\hline$q$ & Number of evaluators \\
\hline$r$ & Number of criteria \\
\hline$w_{1, \alpha, \beta}$ & The weight that shows the importance of the evaluation made by evaluator $\beta$ for criterion $\alpha$ \\
\hline$w_{2, \alpha}$ & The weight that shows the importance of criterion $\alpha$ in the performance evaluation process \\
\hline$w_{3, \alpha}$ & The weight that shows the importance of criterion $\alpha$ in the overall risk evaluation process \\
\hline$s_{i, \alpha, \beta}$ & The score of one single device of type $i$ sold by supplier $j$ given by evaluator $\beta$ for criterion $\alpha$ \\
\hline
\end{tabular}


Table 2. Input data in the Multi-Objective optimization model for supplier selection

\begin{tabular}{|l|l|}
\hline Symbol & Description \\
\hline$n$ & Number of devices to be purchased \\
\hline$m$ & Number of suppliers \\
\hline$M$ & The sum to be invested (in Euro) (parameter) \\
\hline$W$ & The lower limit for the performance of the portfolio of devices (user parameter) \\
\hline$\rho_{i, j}$ & The selection risk for devices of type $i$ sold by supplier $j$ (obtained in stage 1 ) \\
\hline$p_{i, j}$ & The performance of devices of type $i$ sold by supplier $j$ (obtained in stage 1) \\
\hline$c_{i, j}$ & The cost of one single device of type $i$ at the supplier $j$ \\
\hline$d_{i, j}$ & The maximum number of devices of type $i$ available for selling at supplier $j$ \\
\hline$a_{i}$ & The minimum number of devices of type $i$ that should be bought \\
\hline$b_{i}$ & The maximum number of devices of type $i$ that should be bought \\
\hline
\end{tabular}

$j=1,2, \ldots, m$. The array $x_{i j}$ represents the number of devices of type $i$ bought from supplier $j$.

Suppose that the manager of a company wants to buy a portfolio of $n$ devices from $m$ suppliers so that the selection risk related with the portfolio of devices should be minimized and the performance of the respective portfolio should be maximized. To solve this problem a Multi-Objective model for supplier selection is formulated:

$$
\left\{\begin{array}{c}
\min \left[\sum_{i=1}^{n} \sum_{j=1}^{m} \rho_{i, j} x_{i, j}\right] \\
\max \left[\sum_{i=1}^{n} \sum_{j=1}^{m} p_{i, j} x_{i, j}\right] \\
\sum_{i=1}^{n} \sum_{j=1}^{m} c_{i, j} x_{i, j} \leq M \\
a_{i} \leq \sum_{j=1}^{m} x_{i, j} \leq b_{i}, i=1,2, \ldots n \\
0 \leq x_{i, j} \leq d_{i, j} \\
x_{i, j} \in \mathbb{N}, i=1,2, \ldots, n \quad j=1,2, \ldots, m
\end{array}\right.
$$

Here by $\mathbb{N}$ is denoted the set of natural numbers i.e. the set of non-negative integer numbers. Based on model (5) a minimum risk model is formulated. In the framework of this model the manager wants to find a portfolio of devices that minimizes the selection risk related to the devices involved, which are subject to:

- budget constraints;

- demand constraints;

- constraints related to the overall performance of the devices.

$$
\left\{\begin{array}{c}
\min \left[\sum_{i=1}^{n} \sum_{j=1}^{m} \rho_{i, j} x_{i, j}\right] \\
\sum_{i=1}^{n} \sum_{j=1}^{m} p_{i, j} x_{i, j} \geq W \\
\sum_{i=1}^{n} \sum_{j=1}^{m} c_{i, j} x_{i, j} \leq M \\
a_{i} \leq \sum_{j=1}^{m} x_{i, j} \leq b_{i}, i=1,2, \ldots, n \\
0 \leq x_{i, j} \leq d_{i, j} \\
x_{i, j} \in \mathbb{N}, i=1,2, \ldots, n \quad j=1,2, \ldots, m
\end{array}\right.
$$

For determining the range of parameter $\mathrm{M}$ two optimization models are formulated.

Denote by $M_{1}$ the optimal value for the following problem:

$$
\left\{\begin{array}{c}
\min \left[\sum_{i=1}^{n} \sum_{j=1}^{m} c_{i, j} x_{i, j}\right] \\
a_{i} \leq \sum_{j=1}^{m} x_{i, j} \leq b_{i}, i=1,2, \ldots, n \\
0 \leq x_{i, j} \leq d_{i, j} \\
x_{i, j} \grave{o} N, \quad i=1,2, \ldots, n, j=1,2, \ldots, m
\end{array}\right.
$$

Denote by $M_{2}$ the optimal value for the following problem:

$$
\left\{\begin{array}{c}
\max \left[\sum_{i=1}^{n} \sum_{j=1}^{m} c_{i, j} x_{i, j}\right] \\
a_{i} \leq \sum_{j=1}^{m} x_{i, j} \leq b_{i}, \quad i=1,2, \ldots, n \\
0 \leq x_{i, j} \leq d_{i, j} \\
x_{i, j} \grave{o} N, \quad i=1,2, \ldots, n, \quad j=1,2, \ldots, m
\end{array}\right.
$$


Then the range of parameter $M$ is the interval $\left[M_{1}\right.$, $\left.M_{2}\right]$. For every $M \in\left[M_{1}, M_{2}\right]$ denote by $W_{1}(M)$ the optimal value for the following problem:

$$
\left\{\begin{array}{c}
\min \left[\sum_{i=1}^{n} \sum_{j=1}^{m} p_{i, j} x_{i, j}\right] \\
\sum_{i=1}^{n} \sum_{j=1}^{m} c_{i, j} x_{i, j} \leq M \\
a_{i} \leq \sum_{j=1}^{m} x_{i, j} \leq b_{i}, i=1,2, \ldots, n \\
0 \leq x_{i, j} \leq d_{i, j} \\
x_{i, j} \grave{o} N, i=1,2, \ldots, n, j=1,2, \ldots, m
\end{array}\right.
$$

and by $W_{2}(M)$ the optimal value for the following problem:

$$
\left\{\begin{array}{c}
\max \left[\sum_{i=1}^{n} \sum_{j=1}^{m} p_{i, j} x_{i, j}\right] \\
\sum_{i=1}^{n} \sum_{j=1}^{m} c_{i, j} x_{i, j} \leq M \\
a_{i} \leq \sum_{j=1}^{m} x_{i, j} \leq b_{i}, i=1,2, \ldots, n \\
0 \leq x_{i, j} \leq d_{i, j} \\
x_{i, j} \grave{\mathrm{o} N}, i=1,2, \ldots, n, j=1,2, \ldots, m
\end{array}\right.
$$

Then the range of parameter $W$ is the interval $\left[W_{I}(M), W_{2}(M)\right]$.

\section{Case Study}

Suppose that a manager of a Healthcare Center for geriatrics and gerontology intends to modernize that facility by buying new devices which are fitted with sensors. He wants to buy six types of devices. Denote by $\{$ E1, E2, E3, E4, E5, E6 \} the set of devices which are to be bought. The minimum and maximum number of devices that have to be bought are included in Table 3 .

Table 3. The minimum and maximum number of devices

\begin{tabular}{|c|c|c|c|c|c|c|}
\hline Symbol & $\mathrm{E} 1$ & $\mathrm{E} 2$ & $\mathrm{E} 3$ & $\mathrm{E} 4$ & $\mathrm{E} 5$ & $\mathrm{E} 6$ \\
\hline$a_{i}$ & 15 & 10 & 20 & 25 & 20 & 15 \\
\hline$b_{i}$ & 20 & 15 & 30 & 30 & 30 & 25 \\
\hline
\end{tabular}

A group of three evaluators, that is M1, M2 and M3 was selected for evaluating the devices provided by different suppliers.

The suppliers who are able to deliver the necessary devices were identified and, among them, three suppliers were selected: S1, S2 and S3. The quality criteria for evaluating the devices provided by the aforementioned suppliers were defined, as it is swn in Figure 2. Five performance criteria were taken into consideration: accuracy, trust and security, response time, precision and provider reputation. The respective criteria were denoted by $\mathrm{C} 1, \mathrm{C} 2, \mathrm{C} 3, \mathrm{C} 4$ and $\mathrm{C} 5$.

A set of three weights is obtained by means of a weighting method.

The performance of the devices involved is evaluated by every evaluator in the aforementioned group by assigning scores on a scale from 1 to 10 to each device provided by a certain supplier with regard to each of the above-mentioned criteria. The quality of expertise for each evaluator is taken into account by means of the evaluatorrelated weights. Based on equations (1) and (2) the performance of the devices involved is obtained (see Table 4).

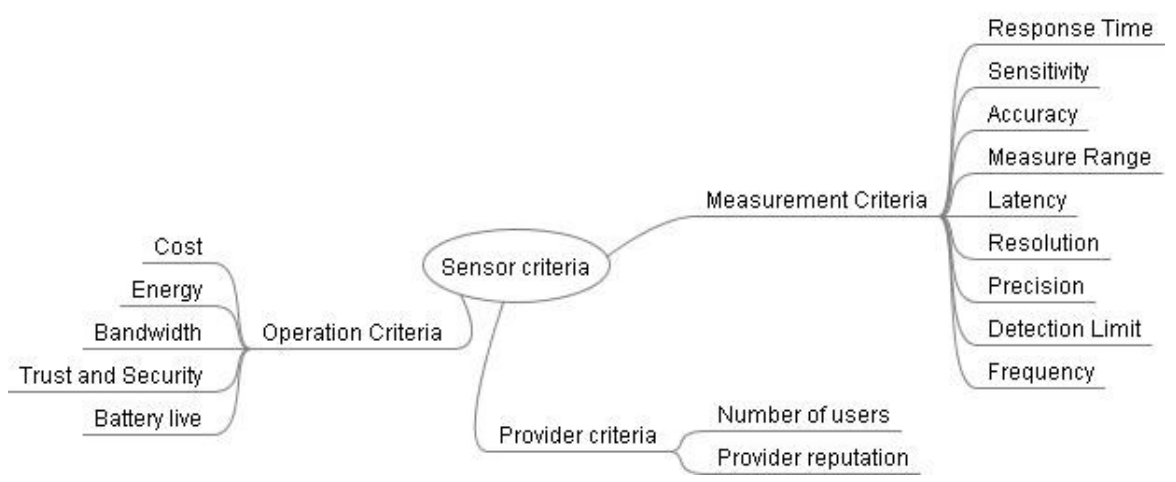

Figure 2. Sensor criteria 
Table 4. The devices' performance

\begin{tabular}{cccc}
\hline \multirow{2}{*}{ Devices } & \multicolumn{3}{c}{ Suppliers } \\
\cline { 2 - 4 } & S1 & S2 & S3 \\
\hline E1 & 8.895 & 8.91 & 8.895 \\
E2 & 8.915 & 8.935 & 8.92 \\
E3 & 8.91 & 8.905 & 8.89 \\
E4 & 8.915 & 8.89 & 8.915 \\
E5 & 8.945 & 8.945 & 8.945 \\
E6 & 8.93 & 8.89 & 8.875 \\
\hline
\end{tabular}

Based on equations (3) and (4) the selection risk related to the aforementioned devices was obtained (see Table 5).

Table 5. The selection risk related to the aforementioned devices

\begin{tabular}{cccc}
\hline \multirow{2}{*}{ Devices } & \multicolumn{3}{c}{ Suppliers } \\
\cline { 2 - 4 } & S1 & S2 & S3 \\
\hline E1 & 0.115625 & 0.075625 & 0.115625 \\
E2 & 0.086875 & 0.081875 & 0.089375 \\
E3 & 0.11375 & 0.10625 & 0.1 \\
E4 & 0.108125 & 0.113125 & 0.118125 \\
E5 & 0.084375 & 0.085625 & 0.073125 \\
E6 & 0.10125 & 0.13625 & 0.09625 \\
\hline
\end{tabular}

The vectors containing the minimum/maximum number of pieces of devices and the matrix of devices costs are build.

In order to determine the range of parameter $M$ the models (7) and (8) are solved and the parameters $M_{1}$ and $M_{2}$ were obtained. $M_{1}=8720$ is the optimal value for the objective function of the problem (7) and $M_{2}=12810$ is the optimal value for the problem (8). Thus the range of parameter $M$ is the interval [8720, 12810]. Suppose that the manager chooses $M=12401$
Euro. It is obvious that the value of parameter $M$ belongs the above interval.

For this value of parameter $M$, we calculate the range of parameter $W$. Models (9) and (10) were solved in order to obtain $W_{1}$ and $W_{2} . W_{1}=934.65$ is the optimal objective function for the problem (9) and $W_{2}=1338.58$ is the optimal value for the problem (10). Thus the range of parameter $W$ is the interval [934.65,1338.58]. The manager chooses $W=1139.05$. It's obvious that the value of parameter $W$ belongs to this interval.

The model (6) is solved for parameters $M=12401$ and $W=1139.05$. The optimal solution is presented in Table 6.

Table 6. The optimal solution for parameter $M=$ 12401 and $W=1139.05$

\begin{tabular}{|l|c|c|c|c|c|c|}
\hline & \multicolumn{4}{|c|}{ Supplier S3 } & \multicolumn{2}{c|}{ Supplier S2 } \\
\hline Devices & E1 & E2 & E3 & E4 & E5 & E6 \\
\hline Number of pieces & 20 & 10 & 22 & 30 & 20 & 25 \\
\hline
\end{tabular}

\section{Sensitivity Analysis}

A sensitivity analysis will be performed for the problem presented in this case study by varying the values of parameters $M$ and $W$.

For every $M \in[8720,12810]$ the range of parameter $W$ was calculated. The value of parameter $M$ starts from 8720 and increases with the step $(12810-8720) / \mathrm{IN}$ to the limit of the range $M=12810$. IN is the number of iterations: $\mathrm{IN}=20$ in this case study.

The models (9) and (10) were solved for each value of $M$ in order to obtain the parameters $W_{1}$ and $W_{2}$. The ranges of parameter $W$ for 5 selected values of parameter $M$ are presented in Table 7 and Figure 3.

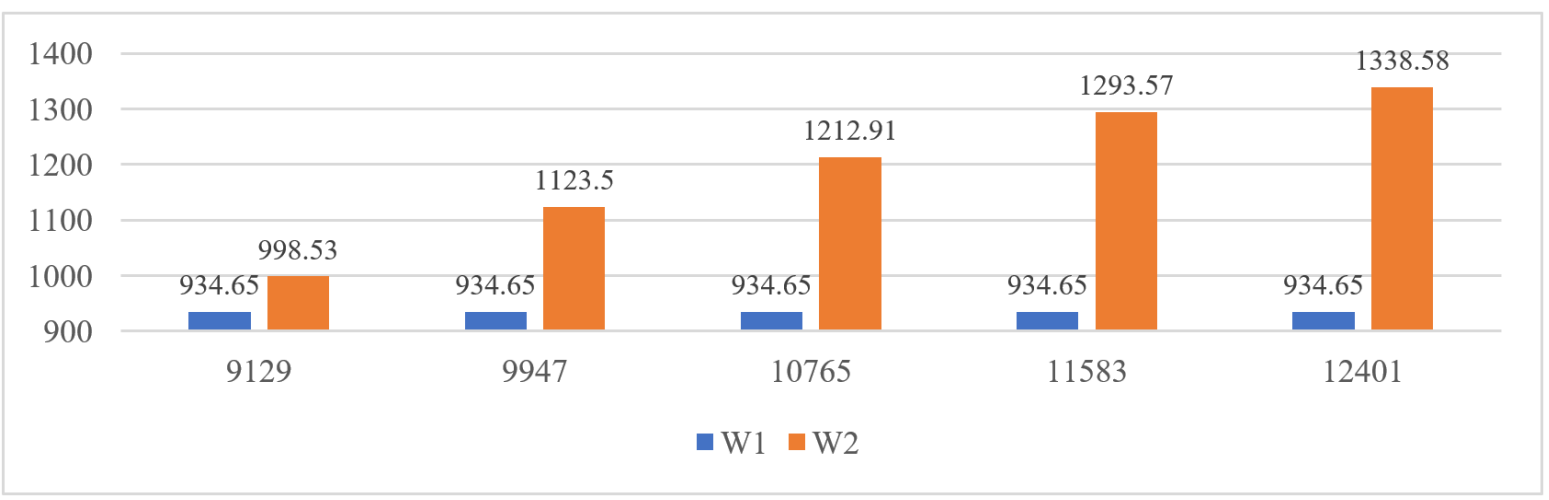

Figure 3. The range of parameter $W$ for different values of $M$ in the interval [8720, 12810] 
Table 7. The range of parameter $W$ for diferent values of parameter $M$ in the interval $[8720,12810]$

\begin{tabular}{|l|r|r|r|r|r|}
\hline $\boldsymbol{M}=$ & $\mathbf{9 1 2 9}$ & $\mathbf{9 9 4 7}$ & $\mathbf{1 0 7 6 5}$ & $\mathbf{1 1 5 8 3}$ & $\mathbf{1 2 4 0 1}$ \\
\hline $\boldsymbol{W} \mathbf{1}$ & 934.65 & 934.65 & 934.65 & 934.65 & 934.65 \\
$\boldsymbol{W} \mathbf{2}$ & 998.53 & 1123.5 & 1212.91 & 1293.57 & 1338.58 \\
\hline
\end{tabular}

The model (6) is solved by varying the value of parameter $M$ in the range [8720,12810] and by varying the value of parameter $W$ in the range calculated as a function of $M$. The values of the related objective function are presented in Table 8 and the related efficient frontier is illustrated in Figure 4.

Solutions: Sol1, Sol2, ..., Sol8 obtained by implementing the Multi-Objective model (6) are presented in Table 9 for parameter $M=12401$ and parameter $W$ for the interval $[934.65 ; 1338.58]$.

On the first position of the column related to the optimal solution Soll (the third column of Table 9) one can notice number 15 which is the number of devices E1 that should be bought from supplier S3. On the second position there is number 10 which is the number of devices E2, that should be bought from supplier S1, etc.

When the values of parameter $W$ increase the number of devices increases too. Thus, for $W=934.65$ the manager can buy a number of 105 devices and for $W=1330.68$ the manager can buy a number of 149 devices.

The solver employed for solving the abovementioned models is a MIP one from GAMS.
Table 8. The values of the objective function for different values of $W$ and $M$

\begin{tabular}{cccccc}
\hline $\boldsymbol{W}=$ & \multicolumn{5}{c}{$\boldsymbol{M}=$} \\
\cline { 2 - 6 } & $\mathbf{9 1 2 9}$ & $\mathbf{9 9 4 7}$ & $\mathbf{1 0 7 6 5}$ & $\mathbf{1 1 5 8 3}$ & $\mathbf{1 2 4 0 1}$ \\
\hline $\mathbf{9 3 4 . 6 5}$ & 10.59 & 10.59 & 10.59 & 10.59 & 10.59 \\
$\mathbf{9 4 7 . 4 3}$ & 11.75 & 10.59 & 11.75 & 11.75 & 11.75 \\
$\mathbf{9 6 0 . 2}$ & 11.88 & 11.75 & 11.89 & 11.89 & 11.89 \\
$\mathbf{9 7 2 . 9 7}$ & 12.11 & 11.89 & 12.16 & 12.16 & 12.16 \\
$\mathbf{9 8 5 . 7 5}$ & 12.11 & 12.16 & 12.29 & 12.29 & 12.29 \\
$\mathbf{9 9 8 . 5 2}$ & 12.11 & 12.29 & 12.57 & 12.57 & 12.57 \\
$\mathbf{1 0 1 1 . 3}$ & & 12.57 & 12.7 & 12.7 & 12.7 \\
$\mathbf{1 0 2 4 . 0 7}$ & & 12.7 & 12.84 & 12.84 & 12.84 \\
$\mathbf{1 0 3 6 . 8 5}$ & & 12.84 & 13.09 & 13.09 & 13.09 \\
$\mathbf{1 0 4 9 . 6 3}$ & & 13.09 & 13.21 & 13.21 & 13.21 \\
$\mathbf{1 0 6 2 . 4}$ & & 13.21 & 13.45 & 13.45 & 13.45 \\
$\mathbf{1 0 7 5 . 1 8}$ & & 13.44 & 13.57 & 13.57 & 13.57 \\
$\mathbf{1 0 8 7 . 9 5}$ & & 13.54 & 13.8 & 13.8 & 13.8 \\
$\mathbf{1 1 0 0 . 7 3}$ & & 13.77 & 13.91 & 13.91 & 13.91 \\
$\mathbf{1 1 1 3 . 5}$ & & 13.84 & 14.03 & 14.03 & 14.03 \\
$\mathbf{1 1 2 6 . 2 8}$ & & 13.84 & 14.26 & 14.26 & 14.26 \\
$\mathbf{1 1 3 9 . 0 5}$ & & & 14.37 & 14.37 & 14.37 \\
$\mathbf{1 1 5 1 . 8 3}$ & & & 14.59 & 14.6 & 14.6 \\
$\mathbf{1 1 6 4 . 6}$ & & & 14.71 & 14.71 & 14.71 \\
$\mathbf{1 1 7 7 . 3 8}$ & & & 14.88 & 14.94 & 14.94 \\
$\mathbf{1 1 9 0 . 1 5}$ & & & 14.91 & 15.05 & 15.05 \\
$\mathbf{1 2 0 2 . 9 3}$ & & & 14.91 & 15.17 & 15.17 \\
$\mathbf{1 2 1 5 . 7}$ & & & & 15.37 & 15.37 \\
$\mathbf{1 2 2 8 . 4 8}$ & & & & 15.46 & 15.46 \\
$\mathbf{1 2 4 1 . 2 5}$ & & & & 15.62 & 15.64 \\
$\mathbf{1 2 5 4 . 0 3}$ & & & & 15.68 & 15.73 \\
$\mathbf{1 2 6 6 . 8}$ & & & & 15.76 & 15.9 \\
$\mathbf{1 2 7 9 . 5 8}$ & & & & 15.77 & 15.99 \\
$\mathbf{1 2 9 2 . 3 5}$ & & & & 15.77 & 16.16 \\
$\mathbf{1 3 0 5 . 1 3}$ & & & & & 16.24 \\
$\mathbf{1 3 1 7 . 9}$ & & & & & 16.33 \\
\hline & & & & & \\
\hline
\end{tabular}

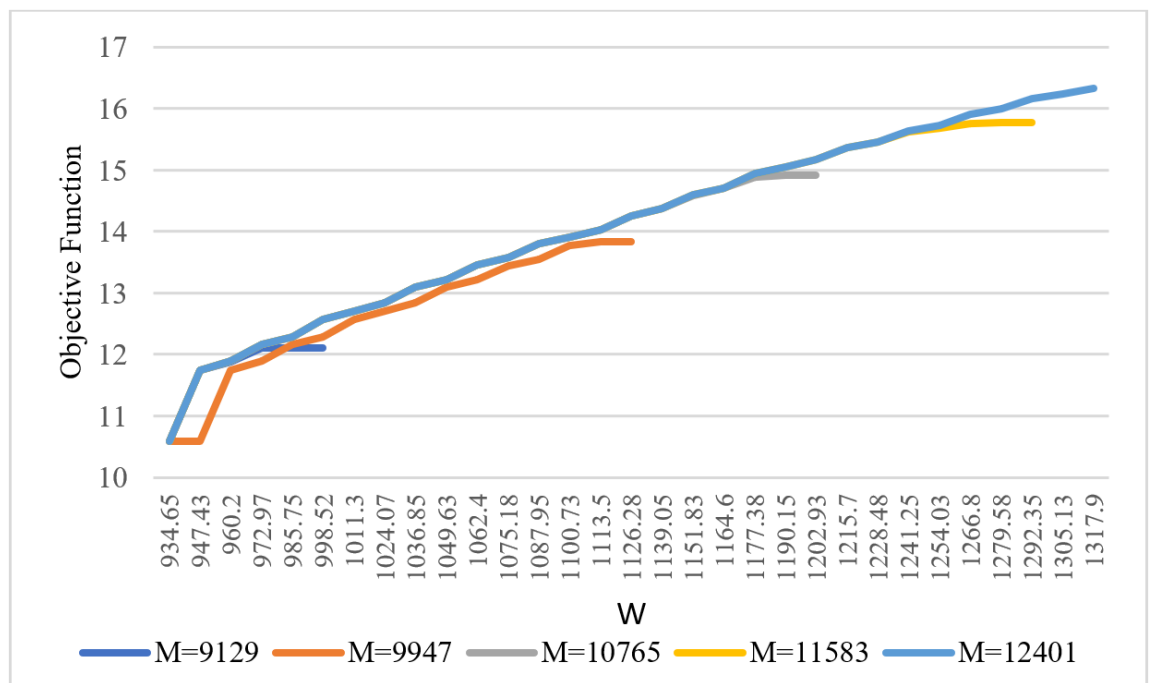

Figure 4. The efficient frontier 
Table 9. Solutions obtained by implementing the Multi Objective model (6)

\begin{tabular}{|c|c|c|c|c|c|c|c|c|c|c|}
\hline Devices & Supplier & Sol1 & Sol2 & Sol3 & Sol4 & Sol5 & Sol6 & Sol7 & Sol 8 & Sol 9 \\
\hline \multirow{2}{*}{ E1 } & S3 & 15 & 15 & 15 & 17 & 20 & 20 & 20 & 20 & 20 \\
\hline \multirow{2}{*}{ E2 } & S1 & 10 & 0 & 0 & 0 & 0 & 0 & 0 & 0 & 0 \\
\cline { 2 - 13 } & S3 & 0 & 10 & 10 & 10 & 10 & 10 & 14 & 15 & 14 \\
\hline \multirow{2}{*}{ E3 } & S1 & 0 & 20 & 20 & 20 & 22 & 28 & 30 & 30 & 30 \\
\cline { 2 - 12 } & $\mathrm{S} 3$ & 20 & 0 & 0 & 0 & 0 & 0 & 0 & 0 & 0 \\
\hline \multirow{2}{*}{ E4 } & S2 & 25 & 0 & 0 & 0 & 0 & 0 & 0 & 0 & 0 \\
\cline { 2 - 12 } & $\mathrm{S} 3$ & 0 & 25 & 26 & 30 & 30 & 30 & 30 & 30 & 30 \\
\hline \multirow{2}{*}{ E5 } & $\mathrm{S} 1$ & 0 & 0 & 0 & 0 & 0 & 0 & 0 & 0 & 6 \\
\cline { 2 - 11 } & $\mathrm{S} 2$ & 20 & 20 & 20 & 20 & 20 & 20 & 20 & 25 & 24 \\
\hline \multirow{2}{*}{ E6 } & $\mathrm{S} 2$ & 0 & 20 & 25 & 25 & 25 & 25 & 25 & 25 & 25 \\
\cline { 2 - 11 } & $\mathrm{S} 3$ & 15 & 0 & 0 & 0 & 0 & 0 & 0 & 0 & 0 \\
\hline
\end{tabular}

\section{Conclusion}

Supplier selection is a complex decision-making problem. It involves weighing several alternatives against multiple conflicting criteria. This problem becomes even more complex when the respective evaluation is performed by several evaluators (decision makers), each of them with his own perception regarding the importance of the aforementioned criteria and the performance of the evaluated alternatives.

This paper presented an original two stages approach to supplier selection. The first stage was based on a group decision model which is meant for calculating the performance of the devices involved and the inherent selection risk. The second stage was based on a Multi-Objective model that took into account the selection risk related to those devices and their performance in the presence of budget and demand constraints. Multi-criteria weighting methods can be used for computing weights related to the devices ' performance and the inherent selection risk.

The group decision approach involved three types of weights: the evaluator-related weights (coefficient of authority), the criteria weights in the device performance evaluation and the criteria weights

\section{REFERENCES}

Baykasoglu, A., Kaplanoglu, V., Durmusoglu, Z. D. U. \& Sahin, C. (2013). Integrating Fuzzy DEMATEL and Fuzzy Hierarchical TOPSIS Methods for Truck Selection, Expert Systems with Applications, 40(3), 899-907.

de Boer, L., Labro, E. \& Morlacchi, P. (2001). A review of methods supporting supplier selection, related to the evaluation of the selection risk for the respective devices. Our approach was employed for analyzing a case study based in a healthcare center.

Finally, it is worth mentioning that, starting from this decision-making approach, a software module that would guide managers in the process of purchasing management could be created. The respective module could be embedded in a decision support system.

\section{Acknowledgements}

The research presented in this paper is supported through the project "vINCI: Clinically-validated INtegrated Support for Assistive Care and Lifestyle Improvement: the Human Link" (which is funded through the EUAAL2017 Programme and Executive Unit for Financing Higher Education, Research, Development and Innovation-UEFISCDI Romania), the project "Non-invasive monitoring and health assessment of the elderly in a smart environment (RO-Smart Ageing)" (funded through the Romanian Core Program of the Ministry of Research and Innovation) and the project "New solutions for complex problems in current ICT research fields based on modelling and optimization" (funded by the Romanian Core Program of the Ministry of Research and Innovation).

\section{European Journal of Purchasing \& Supply Management, 7(2), 75-89.}

Chai, J., Liu J. N. K. \& Ngai, E. W. T. (2013). Application of decision-making techniques in supplier selection: A systematic review of literature, Expert Systems with Applications, 40(10), 3872-3885. 
Chai, J. \& Ngai, E. W. (2020). Decisionmaking techniques in supplier selection: Recent accomplishments and what lies ahead, Expert Systems with Applications, 140, 112903.

Degraeve, Z., Labro, E. \& Roodhooft, F. (2000). An evaluation of supplier selection methods from a Total Cost of Ownership perspective, European Journal of Operational Research, 125(1), 34-59.

Dickson, G. W. (1966). An analysis of vendor selection systems and decision, Journal of Purchasing, 2(15), 1377-1382.

Dupont, L., Bernard, C., Hamdi, F. \& Masmoudi, F. (2018). Supplier selection under risk of delivery failure: A decision-support model considering managers' risk sensitivity, International Journal of Production Research, 56(3), 1054-1069.

Fahimnia, B., Sarkis, J. \& Davarzani, H. (2015). Green supply chain management: A review and bibliometric analysis, International Journal of Production Economics, 162(C), 101-114.

Filip, F. G., Zamfirescu, C. B. \& Ciurea, C. (2017). Computer-Supported Collaborative DecisionMaking. Springer International Publishing.

Ho, W., Xu, X. \& Dey P. K. (2010). Multicriteria decision-making approaches for supplier evaluation and selection: A literature review, European Journal of Operational Research, 202(1), 16-24.

Hu, H., Xiong, H., You, Y. \& Yan, W. (2016). A mixed integer programming model for supplier selection and order allocation problem with fuzzy multi-objective, Scientific Programming, 2016(15), 1-13. Article Number: 9346781, DOI: $10.1155 / 2016 / 9346781$

Kellner, F. \& Utz, S. (2019). Sustainability in supplier selection and order allocation: Combining integer variables with Markowitz portfolio theory, Journal of Cleaner Production, 214, 462-474.

Kersuliene, V., Zavadskas, E. K. \& Turskis, Z. (2010). Selection of rational dispute resolution method by applying new step wise weight assessment ratio analysis (SWARA), Journal of
Business Economics and Management, 11(2), 243-258.

Radulescu, C. Z. \& Radulescu, M. (2018). Group decision support approach for cloud quality of service criteria weighting, Studies in Informatics and Control, 27(3), 275-284. DOI: 10.24846/ v27i3y201803

Rezaei, J. (2015). Best-worst multi-criteria decision-making method, Omega, 53, 49-57.

Saaty, T. L. (1980). The Analytic Hierarchy Process. McGraw-Hill Press, NewYork.

Sodenkamp, M. A., Tavana, M. \& Di Caprio, D. (2016). Modeling synergies in multi-criteria supplier selection and order allocation: An application to commodity trading, European Journal of Operational Research, 254(3), 859-874.

Weber, C. A., Current J. R. \& Benton, W. C. (1991). Vendor selection criteria and methods, European Journal of Operational Research, $50(1), 2-18$.

Wetzstein, A., Hartmann, E., Benton, W. C., Jr. \& Hohenstein, N. O. (2016). A systematic assessment of supplier selection literature State-of-the-art and future scope, International Journal of Production Economics, 182, 304-323.

Zavadskas E. K., Stević Z, Turskis, Z. \& Tomašević, M. (2019). A Novel Extended EDAS in Minkowski Space (EDAS-M) Method for Evaluating Autonomous Vehicles, Studies in Informatics and Control, 28(3), 255-264. DOI: $10.24846 / \mathrm{v} 28 \mathrm{i} 3 \mathrm{y} 201902$

Zimmer, K., Fröhling, M. \& Schultmann, F. (2016). Sustainable supplier management A review of models supporting sustainable supplier selection, monitoring and development, International Journal of Production Research, 54(5), 1412-1442.

Zolfani, S., Yazdani, M. \& Zavadskas, E. (2018). An extended Stepwise Weight Assessment Ratio Analysis (SWARA) method for improving criteria prioritization process, Soft Computing, 22, 73997405. DOI: 10.1007/s00500-018-3092-2 